\title{
A HOPF MODULE CHARACTERIZATION OF HOPF ALGEBRAS
}

\author{
DAVID E. RADFORD ${ }^{1}$
}

\begin{abstract}
A bialgebra over a field is a Hopf algebra if and only if all (nonzero) right Hopf modules are free, as modules, on a set of invariants.
\end{abstract}

0. Introduction. Let $A$ be a Hopf algebra over a field $k$. The fact that any (nonzero) right $A$-Hopf module $M$ is free as an $A$-module on any linear basis of the invariants of $M$ is of profound importance in the theory of Hopf algebras [S, Theorem 4.1.1]. In this paper we prove a converse-we show that Hopf algebras over $k$ are those bialgebras over $k$ whose (nonzero) right Hopf modules are free as a module on a set of invariants. Thus the converse characterizes Hopf algebras over a field in terms of Hopf modules.

Actually we show that for a bialgebra $A$ over $k$ to be a Hopf algebra, it is sufficient to know that the (nonzero) finitely generated right $A$-Hopf modules, which are free as an $A$-module, have a module basis of invariants. Thus what distinguishes bialgebras from Hopf algebras is not so much a matter of Hopf modules being free but rather a matter of free Hopf modules having a certain type of basis.

1. Preliminaries. Let $C$ be a coalgebra over a field $k$, and suppose $(M, \omega)$ is a finite-dimensional right $C$-comodule. Let $m_{1}, \ldots, m_{s}$ be a basis for $M$ and let $e_{i j} \in C$ for $1 \leqslant i, j \leqslant s$ be defined by $\omega\left(m_{j}\right)=\sum_{i} m_{i} \otimes e_{i j} \in M \otimes_{k} C$. Then the equations

$$
\Delta e_{i j}=\sum_{k} e_{i k} \otimes e_{k j}
$$

and

$$
\varepsilon\left(e_{i j}\right)=\delta_{i j} \text { for } 1 \leqslant i, j \leqslant s
$$

follow from the equations $\omega \otimes I \circ \omega=I \otimes \Delta \circ \omega$ and $I \otimes \varepsilon \circ \omega=I$ respectively.

Now suppose $A$ is a bialgebra over $k$ and $(M, \omega)$ is any right $A$-comodule. Write $\omega(m)=\sum \underline{m}_{(1)} \otimes m_{(2)} \in M \otimes_{k} A$ for $m \in M$. Then $\left(M \otimes_{k} A, \omega_{A}\right)$ is a right $A$ comodule where $\omega_{A}(m \otimes a)=\sum\left(\underline{m}_{(1)} \otimes a_{(1)}\right) \otimes m_{(2)} a_{(2)}$ for $m \in M$ and $a \in A$. Regarding $M \otimes_{k} A$ as a right $A$-module by extension of scalars $((m \otimes a) \circ b=m \otimes a b)$ it is easy to check that $M \otimes_{k} A$ is a right $A$-Hopf module. Note that $M \otimes_{k} A$ is free as an $A$-module if $M \neq(0)$.

Received by the editors July 26, 1982.

1980 Mathematics Subject Classification. Primary 16A24; Secondary 16A36, 16A64.

Key words and phrases. Hopf algebra, category of Hopf modules.

'Research supported in part by National Science Foundation grant NSF MCS-8201481.

(C)1983 American Mathematical Society $0002-9939 / 82 / 0000-0833 / \$ 01.50$ 
For any right $A$-comodule $M$ we say $m \in M$ is an invariant if $\omega(m)=m \otimes 1$ and let $M_{f} \subseteq M$ denote the subspace of invariants of $M$. The purpose of this section is to prove the following.

LemMa. Let $A$ be a bialgebra over a field $k$, and suppose $(M, \omega)$ is a finite-dimensional right $A$-comodule. Assume further $\left(M \otimes_{k} A, \omega_{A}\right)$ has a module basis of invariants. Then if $m_{1}, \ldots, m_{s}$ is a linear basis for $M$, the $s \times s$ matrix $\left(e_{i j}\right) \in M_{s}(A)$ is invertible, where $e_{i j}$ is defined by $\omega\left(m_{j}\right)=\sum_{i} m_{i} \otimes e_{i j}$.

Proof. Let $x=\Sigma_{j i} m_{j} \otimes a_{j} \in M \otimes_{k} A$. Then by definition

$$
\omega_{A}(x)=\sum_{j, k}\left(m_{k} \otimes a_{j(1)}\right) \otimes e_{k j} a_{j(2)},
$$

so $x$ is invariant if and only if $\sum_{j} a_{j(1)} \otimes e_{k j} a_{j(2)}=a_{k} \otimes 1$ for $1 \leqslant k \leqslant s$.

Note that $m_{1} \otimes 1, \ldots, m_{s} \otimes 1$ is a module basis for $M \otimes_{k} A$. Since ker $\varepsilon$ is a cofinite ideal of $A$, any module basis has $s$ elements. Let $x_{1}, \ldots, x_{s} \in\left(M \otimes_{k} A\right)$, be a module basis for $M \otimes_{k} A$, and write $x_{i}=\Sigma_{j} m_{j} \otimes a_{j i}$ where $a_{j i} \in A$. Since $x_{i}$ is invariant it follows $\sum_{j} a_{j i(1)} \otimes e_{k j} a_{j i(2)}=a_{k i} \otimes 1$ for $1 \leqslant k \leqslant s$. Applying $\varepsilon \otimes I$ to this equation we deduce

$$
\sum_{j} e_{k j} a_{j i}=\varepsilon\left(a_{k i}\right) 1 \text { for } 1 \leqslant i, k \leqslant s .
$$

Observe $x_{j}=\Sigma_{i}\left(m_{i} \otimes 1\right) a_{i j}$ means that the $s \times s$ matrix $\left(a_{i j}\right) \in M_{s}(A)$ is a change of basis matrix, hence is invertible. Therefore $\left(\varepsilon\left(a_{i j}\right) 1\right)$ is invertible, so by 1.3 $\left(e_{i j}\right)$ is the product of invertible matrices. Q.E.D.

2. The main theorem. We characterize those bialgebras over a field which are Hopf algebras.

THEOREM. Let $A$ be a bialgebra over a field $k$. Then the following are equivalent:

(a) Any (nonzero) right A-Hopf module is free, as a module, and any linear basis of invariants is a module basis.

(b) Any (nonzero) finitely generated right A-Hopf module is free, as a module, and has a module basis consisting of invariants.

(c) Any (nonzero) right A-Hopf module, which is finitely generated and free as an A-module, has a module basis consisting of invariants.

(d) $A$ is a Hopf algebra.

Proof. (d) $\Rightarrow$ (a) is [S, Theorem 4.1.1]. (a) $\Rightarrow$ (b) and (b) $\Rightarrow$ (c) are trivial. Thus it suffices to show $(c) \Rightarrow(d)$.

Assume the hypothesis of (c), and let $C \subseteq A$ be a finite dimensional subcoalgebra. To show that $A$ is a Hopf algebra, by [R, Lemma 2(b)] it suffices to show that the inclusion $\iota: C \rightarrow A$ has an inverse in the convolution algebra $\operatorname{Hom}_{k}(C, A)$, that is there exists a linear map $s: C \rightarrow A$ satisfying

$$
\begin{aligned}
s * \imath(c) & =\sum s\left(c_{(1)}\right) c_{(2)}=\varepsilon(c) 1 \\
& =\sum c_{(1)} s\left(c_{(2)}\right)=\iota * s(c) \quad \text { for } c \in C .
\end{aligned}
$$


Regard $M=C$ as a right $A$-comodule, and choose a linear basis $m_{1}, \ldots, m_{s}$ for $M$ satisfying $\varepsilon\left(m_{i}\right)=\delta_{1 i}$ for $1 \leqslant i \leqslant s$. Let $e_{i j} \in A$ be defined by $\Delta m_{j}=\sum_{i} m_{i} \otimes e_{i j}$. Observe that $e_{i j} \in C_{1 i}$ since $M$ is in fact a coalgebra, and that $m_{j}=\Sigma_{i} \varepsilon\left(m_{i}\right) e_{1 j}=e_{1 j}$ for $1 \leqslant j \leqslant s$.

By the lemma $\left(e_{i j}\right) \in M_{s}(A)$ has an inverse $\left(a_{i j}\right)$. Define a linear map $s: C \rightarrow A$ by $s\left(e_{1 j}\right)=a_{1 j}$ for $1 \leqslant j \leqslant s$. Then the computation

$$
s * \iota\left(e_{1 i}\right)=\sum_{j} a_{1 j} e_{j i}=\delta_{1 i} 1=\varepsilon\left(e_{1 i}\right) 1
$$

shows that $s * \imath=\eta \circ \varepsilon$, or equivalently $\left(s\left(e_{i j}\right)\right) \in M_{s}(A)$ is a left inverse of $\left(e_{i j}\right)$. Hence $\left(s\left(e_{i j}\right)\right)=\left(a_{i j}\right)$, so $\left(s\left(e_{i j}\right)\right)$ is a right inverse of $\left(e_{i j}\right)$, or equivalently $\iota * s=$ $\eta \circ \varepsilon$. Q.E.D.

As one may guess, "right" may be replaced by "left" in the Theorem. To see this

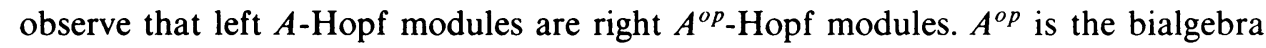
obtained from $A$ by twisting multiplication and comultiplication, specifically $m^{o p}=$ $m \circ T$ and $\Delta^{o p}=T \circ \Delta$ where $T: A \otimes_{k} A \rightarrow A \otimes_{k} A(a \otimes b \rightarrow b \otimes a)$ is the twist map.

\section{REFERENCES}

[R] D. E. Radford, On bialgebras which are simple Hopf modules, Proc. Amer. Math. Soc. 80 (1980). $563-568$.

[S] M. E. Sweedler, Hopf algebras, Benjamin, New York, 1969.

Department of Mathematics, University of Illinois at Chicago Circle, Chicago, Illinois 60680 